\title{
Two Cases of Transhepatic Implantation of Cardiac Implantable Electronic Device: All Roads lead to Rome
}

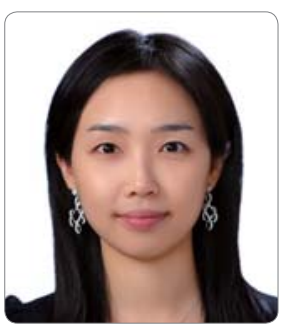

Myung-Jin Cha
Myung-Jin Cha, MD1'; Jae-Sun Uhm, MD, PhD1; Tae-Hoon Kim, MD1; Eue-Keun Choi, MD2; Boyoung Joung, MD, PhD1; Hui-Nam Pak, MD, PhD1; Seil Oh, MD, PhD²; Moon-Hyoung Lee, MD, PhD 1

${ }^{1}$ Division of Cardiology, Severance Cardiovascular Hospital, Yonsei University College of Medicine, Seoul, Republic of Korea

${ }^{2}$ Division of Cardiology, Department of Internal Medicine, Seoul National University Hospital, Seoul, Republic of Korea

Received: November 13, 2017

Revision received: November 21, 2017

Accepted: Nobember 22, 2017

Correspondence: Moon-Hyoung Lee, MD PhD Division of Cardiology, Severance Cardiovascular Hospital, Yonsei University College of Medicine

50-1 Yonsei-ro Seodaemun-gu, Seoul 03722, Republic of Korea

Tel: +82-2-2228-8441, Fax: +82-2-2227-7732

E-mail:mhlee@yuhs.ac

Copyright (c) 2017 The Official Journal of Korean Heart Rhythm Society Editorial Board and EUM \&

Communications

\begin{abstract}
Lead insertion for cardiac implantable electronic devices requires venous access into the right side of the heart. The access route commonly used is from the axillary vein, through the subclavian vein and the superior vena cava. However, in patients with congenital heart malformations or those with vascular stenosis, and/or those who have undergone previous cardiac surgery, the passage of leads might be difficult, and the implantation procedure would show restricted scope. In such cases, insertion of leads through the hepatic vein is known to be a safe procedure. We report 2 cases of patients with limited vascular access who underwent lead implantation using the transhepatic approach-1 patient who underwent placement of an implantable cardioverter defibrillator and the other who underwent placement of a permanent pacemaker.
\end{abstract}

Key Words: - Implantable Cardioverter-Defibrillator - Pacemaker

- Transhepatic

\section{Introduction}

Implantation of a cardiac implantable electronic device (CIED) requires vascular access into the cardiac chambers, and usually a systemic central venous access system via the superior vena cava (SVC) into the right atrium and the ventricle sequentially is used. If no direct access via the SVC is possible, CIED lead placement can be performed epicardially; however, this approach requires an invasive thoracotomy and shows a high threshold and amplitude. Additionally, magnetic resonance-compatible leads are currently unavailable. Transhepatic access for CIED implantation can be a useful alternative route to obtain access to the heart and vascular structures in patients with limited central venous access. We describe 2 patients in whom the SVC approach for lead implantation could not be used and who therefore underwent implantable cardioverter-defibrillator (ICD) placement or permanent pacemaker implantation via the transhepatic route.

\section{Case 1: Transhepatic ICD implantation}

A 60-year-old man was brought to the emergency department for sudden collapse and loss of consciousness during hemodialysis 


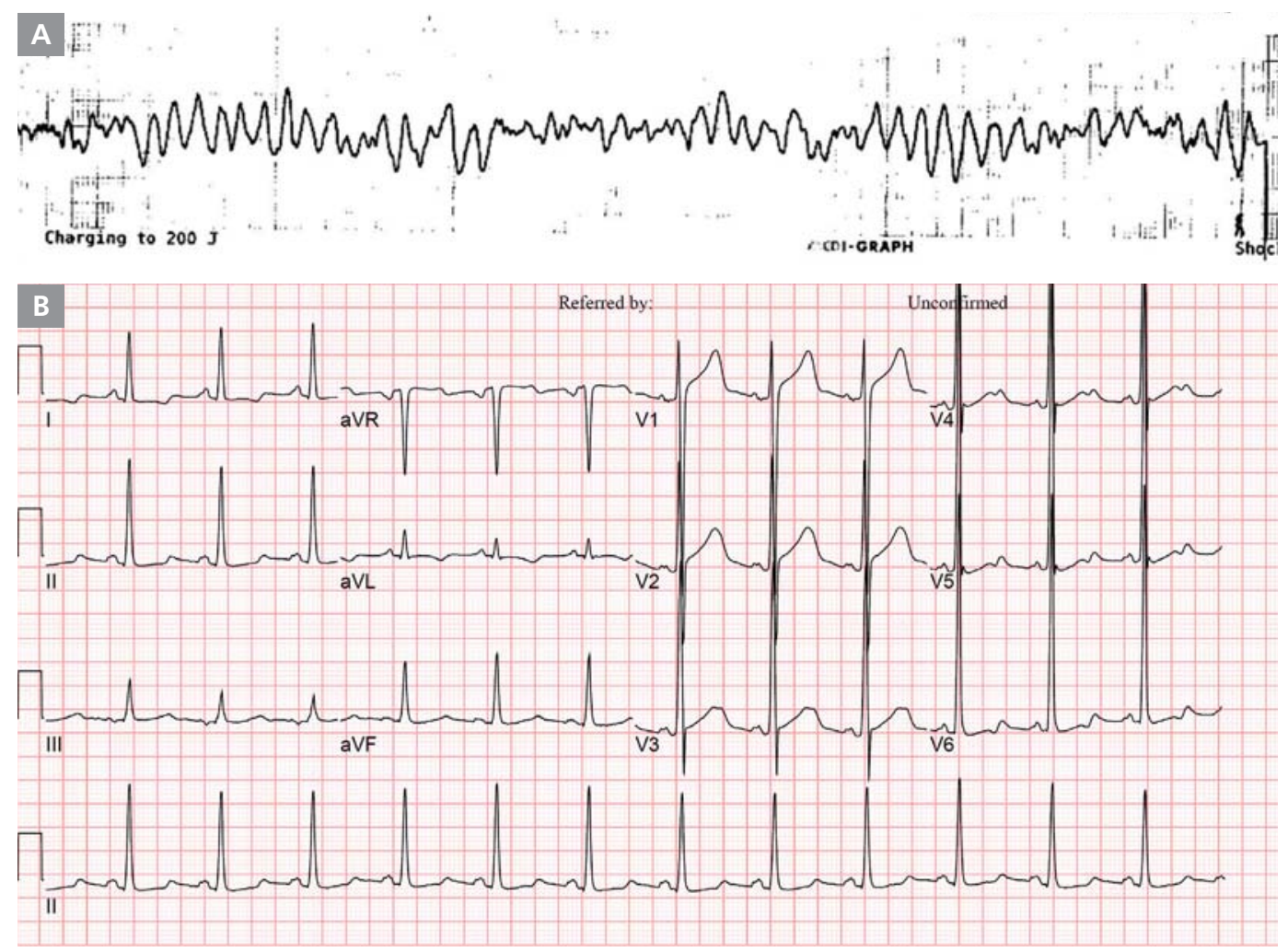

Figure 1. (A) Torsades de pointes in electrocardiography at the time of collapse. (B) QT prolongation (QTc $568 \mathrm{msec}$ ) in electrocardiography 10 days after the event.

performed at another hospital. He was known to have undergone hemodialysis over 16 years through a right arm arteriovenous fistula for end-stage renal disease. Electrocardiography (ECG) performed immediately after his episode of sudden collapse showed torsades de pointes (Figure 1A), and emergency defibrillation was performed by the attending nephrologist. After defibrillation, he showed recovery of his mental status and sinus rhythm. ECG performed at the emergency department showed a normal sinus rhythm, left ventricular hypertrophy, and QT prolongation (corrected QT interval [QTc] $543 \mathrm{~ms}$ ). Serum levels of potassium, ionized calcium, and magnesium were within the reference range $(4.6 \mathrm{mmol} / \mathrm{L}, 4.42 \mathrm{mg} / \mathrm{dL}$, and $1.36 \mathrm{mg} / \mathrm{dL}$, respectively). Echocardiography revealed severely decreased left ventricular systolic function (ejection fraction $21 \%$ ), global hypokinesia, and left ventricular hypertrophy. Coronary angiography revealed intermediate coronary artery disease (50\% stenosis of the mid right coronary artery) and a negative vasospasm provocation test. After the patient was treated using supportive care and hemodialysis over 10 days, his general physical condition showed complete recovery. ECG performed 10 days after the event also showed QTc of $568 \mathrm{~ms}$ (Figure 1B). Follow-up echocardiography showed improved left ventricular systolic function (ejection fraction 48\%). We reviewed his old ECG records. ECGs that had been performed 20 years prior to this presentation also revealed severe QT prolongation (QTc $661 \mathrm{~ms}$ ). During hospitalization, we excluded all potential causes or triggering factors associated with QT prolongation including hypokalemia, hypomagnesemia, hypocalcemia, hypothyroidism, bradyarrhythmias, left ventricular dysfunction, intracranial disease, and drugs that are known to cause QT prolongation. We recommended genetic studies for congenital long QT syndrome; however, he refused to undergo the testing due to the cost factor. He was tentatively diagnosed with congenital long QT syndrome, and we decided to perform ICD implantation in the left pectoral area for secondary prevention of sudden cardiac arrest. However, left arm venography revealed total occlusion of the left subclavian vein and showed the presence of collateral circulation; thus, ICD implantation through the left subclavian vein could not be 

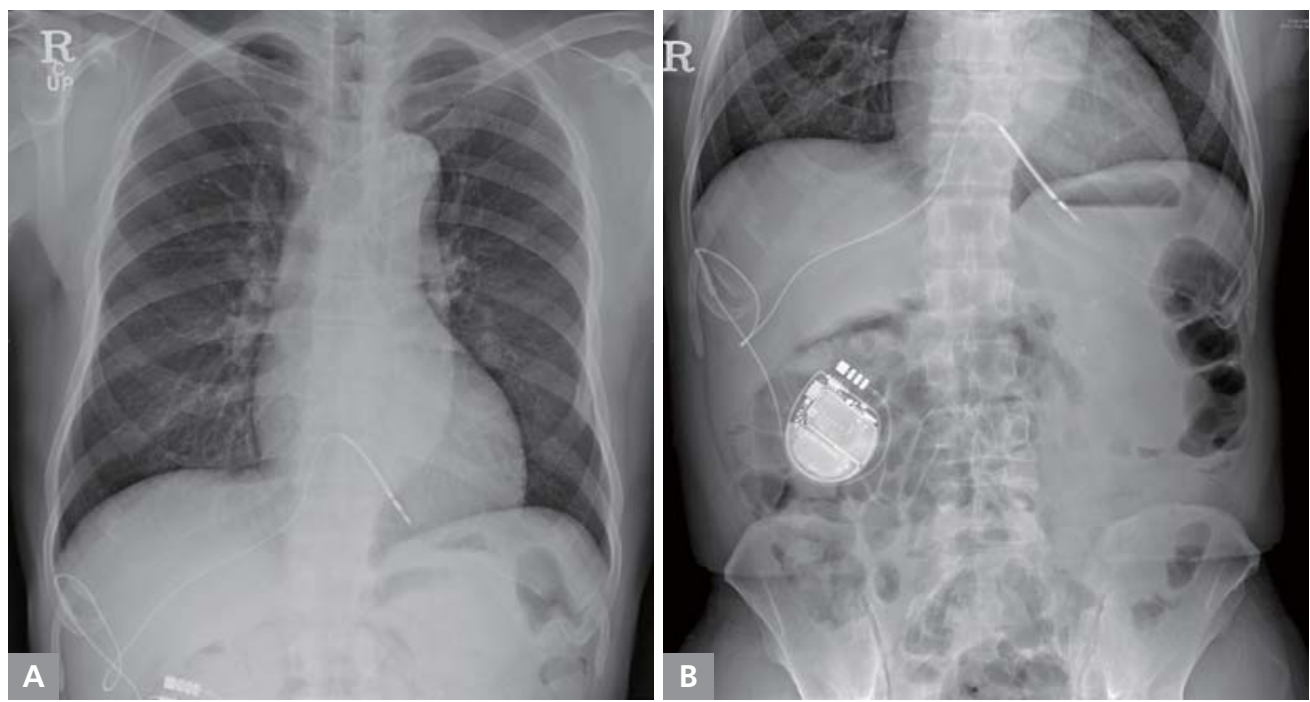

Figure 2. Chest (A) and abdomen (B) X-rays after transhepatic ICD implantation

performed. Moreover, the right subclavian vein needed to be preserved for maintenance of hemodialysis via the right arm arteriovenous fistula. Subcutaneous, epicardial, and transhepatic ICD placement were proposed to the patient as alternative routes for ICD implantation. Because a subcutaneous ICD system was not available in Korea at that time, he would need to wait for the procedure for over a year. He did not wish to delay the ICD implantation, nor did he wish to undergo a sternotomy or thoracotomy for an epicardial ICD implantation. Thus, we decided to implant an ICD via the hepatic vein.

After administration of local anesthesia, the right hepatic vein was punctured using a Chiba needle and approached via the Seldinger technique under ultrasonographic-guidance. A singlecoil defibrillator lead (Durata $7122 \mathrm{Q} / 65 \mathrm{~cm}$, screw type, St. Jude Medical, Valley View Court Sylmar, CA, USA) was inserted into the right ventricle through the hepatic vein and the inferior vena cava and was firmly anchored in the right ventricular apex. The defibrillator lead was connected to the ICD generator (Ellipse VR 1377-36QC, St. Jude Medical). The ICD generator was implanted between the subcutaneous fat and the fascia in the right upper abdomen (Figure 2). ICD interrogation and defibrillation threshold testing showed that the $\mathrm{R}$ wave amplitude, pacing threshold, impedance, and the defibrillation threshold were within an acceptable range $(11.7 \mathrm{mV}, 0.75 \mathrm{~V}$ at $0.4 \mathrm{~ms}, 690$ $\mathrm{ohm}$, and between 20 and $30 \mathrm{~J}$, respectively). Transhepatic ICD implantation was successfully performed in this patient without any complications. The ICD was set as follows: ventricular fibrillation detection cut-off $187 / \mathrm{min}$, tachycardia therapy, antitachycardia pacing during charge $\rightarrow 1$ time of $30 \mathrm{~J} \rightarrow 5$ times of $36 \mathrm{~J}$. Propranolol (60 mg/day) was prescribed for suppression of ventricular tachyarrhythmias. No tachyarrhythmia has been recorded by the ICD over 6 months since the transhepatic ICD implantation has been performed.

\section{Case 2: Transhepatic pacemaker implantation}

A 70-year-old man who was diagnosed with right-sided centraltype small cell lung cancer 6 years prior to presentation reported receiving 4 sessions of chemotherapy and 3 of radiotherapy. However, his disease was observed to progress over 6 years. Chemotherapy was recently discontinued owing to his poor general condition, and his life expectancy was expected to be shorter than a year. He presented to our outpatient clinic with progressive dyspnea (New York Heart Association [NYHA] functional class II to IV). At the outpatient clinic, he suddenly developed syncope and was transferred to the emergency department. His initial ECG showed a complete atrioventricular block with a junctional escape rhythm of $32 \mathrm{bpm}$ (Figure 3). After a temporary pacemaker was inserted via the femoral vein, his dyspnea and general condition showed significant improvement (NYHA class II). There was no medication or laboratory abnormality, which could have caused the complete 


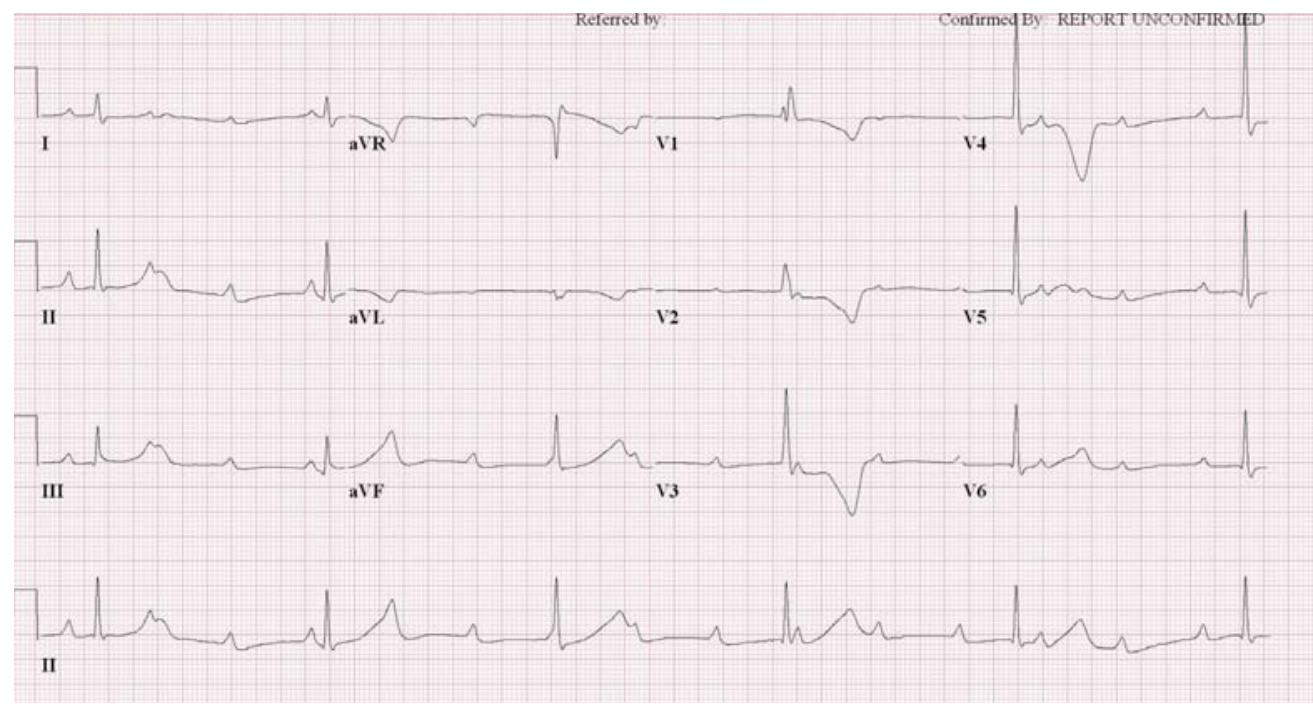

Figure 3. Electrocardiography shows complete atrioventricular block.
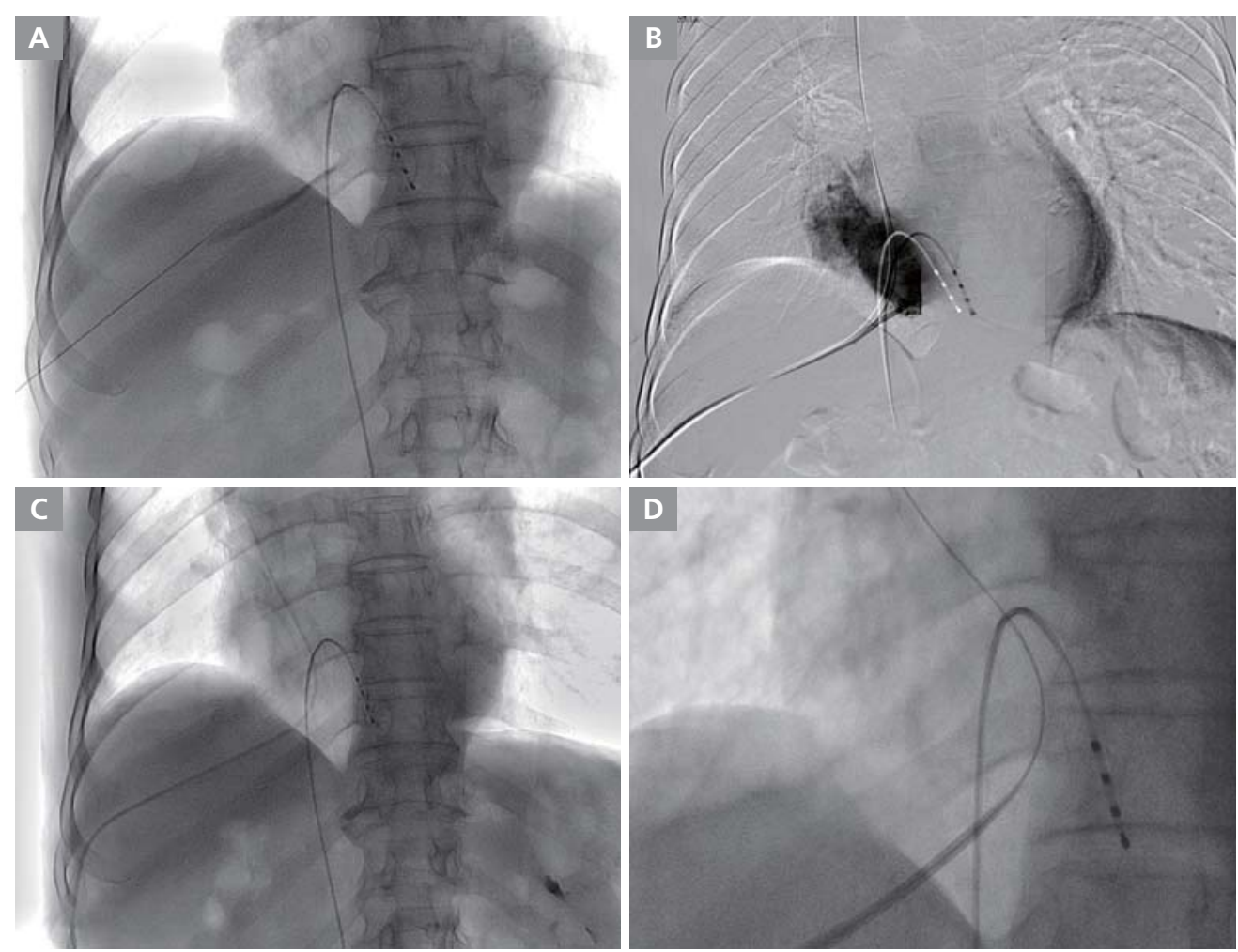

Figure 4. Ultrasonography-guided transhepatic venous approach. (A) Middle hepatic vein drainage confirmed with venography. (B) The right atrium shown by contrast media. (C) A 5-french sheath (12 cm in length) inserted through transhepatic route. (D) The 5-french sheath replaced with 7-french peel-away sheath.

atrioventricular block. His echocardiography showed normal left ventricular function (ejection fraction 60\%) without any regional wall motion abnormality. Although his life expectancy was shorter than a year, we decided to implant a permanent pacemaker because his symptoms showed remarkable improvement with ventricular pacing. We carefully reviewed his computed tomography data because his lung cancer mass was near the right central area, and his initial disease presentation was that of severe SVC syndrome. His primary cancer mass was located at the central area of his right upper lobe, and although the SVC was 


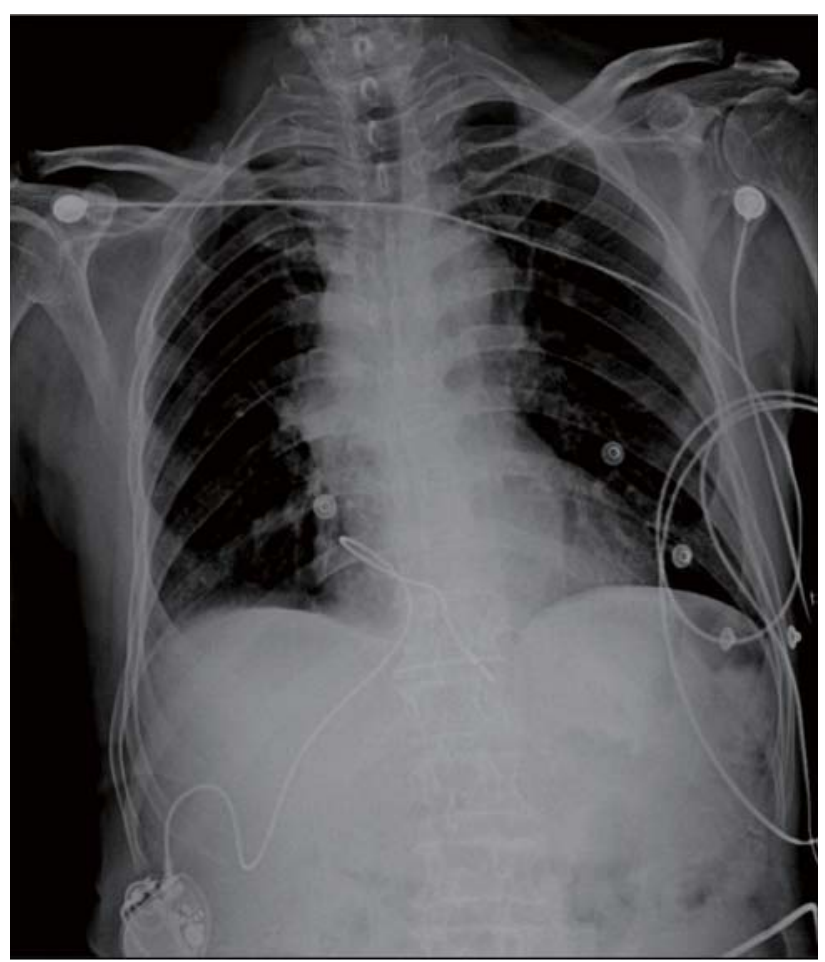

Figure 5. Chest $\mathrm{X}$-ray after transhepatic permanent pacemaker implantation

slightly compressed and narrowed, it was observed to be patent. The patient and his family members were reluctant to use the SVC as a route for lead insertion to avoid a possible complication of SVC syndrome recurrence. Additionally, they refused an epicardial approach due to the invasiveness of the procedure; thus, we decided to use the transhepatic approach via the hepatic vein for pacemaker implantation.

Under ultrasonographic guidance, the middle hepatic vein was punctured by an experienced radiologist, and a 5-French sheath (12 cm in length) was inserted through the hepatic vein. Venography was performed to confirm middle hepatic vein drainage. The detailed sequential procedure has been presented in Figure 4.The screw type lead was curved in the right atrium and inserted into the right ventricular apex to ensure enough redundancy (Figure 5). The pacemaker generator (Endurity MRI 1172, St. Jude Medical, Valley View Court Sylmar, CA, USA) was implanted on the surface of the right $11^{\text {th }}$ and $12^{\text {th }}$ ribs between the subcutaneous fat and the muscle fascia with the following settings: the lead threshold was $0.5 \mathrm{~V}$ at a pulse width of $0.4 \mathrm{~ms}$, the $\mathrm{R}$ wave amplitude was $>12 \mathrm{mV}$, and the impedance was set to $710 \Omega$. His dyspnea was classified as NYHA class II, and he demonstrated a normally functioning pacemaker at his 6-month follow-up, and a chest-abdomen X-ray revealed his electrodes were in a stable position.

\section{Discussion}

We present 2 rare cases describing patients who underwent CIED implantation through a transhepatic approach in whom central venous access was limited/restricted secondary to total occlusion of the subclavian vein or the occurrence of SVC syndrome. To date, no previous case reports describe transhepatic CIED implantation in Korea.

Alternative approaches for pacemaker implantation have been described in patients presenting with limited vascular access routes. Laub et al. have reported transfemoral pacemaker implantation in patients with congenital heart disease. ${ }^{1}$ The transiliac vein approach is a known technique used for implantation of a permanent pacemaker in patients with SVC obstruction after aortic valve re-operation. ${ }^{2}$ Another group has described a large-scale study using the iliac vein for venous entry with leads tunneled into an abdominal pocket at the level of the rectus muscle. ${ }^{3}$ West et al. have described a patient in whom the inferior vena cava was used for venous access via a retroperitoneal approach. ${ }^{4}$ Goktekin et al. have reported a case of permanent pacemaker lead implantation via the azygous vein in a patient with silent SVC syndrome. ${ }^{5}$ Fishberger et al. have reported a patient who underwent CIED implantation using a percutaneous transhepatic approach. ${ }^{6}$

Several limitations and complications have been reported with use of the transhepatic approach (in a previous single-center study describing 124 procedures, 10 major complications have been reported $^{7}$ ): 1) Although several reports describe successful pacemaker implantation in patients via the transhepatic route, ICD implantation via the transhepatic route remains challenging because of concerns regarding effective shock delivery. Cui et al. report successful ICD implantation in a patient post-Glenn or Fontan operation via a percutaneous transhepatic venous approach. ${ }^{8}$ In their study, the defibrillation threshold used for induced ventricular fibrillation was $20 \mathrm{~J}$. In our patient who underwent ICD implantation, the defibrillation threshold was set between 20 and 30 J. 2) The liver is a highly vascular organ; 
therefore, hepatic injury during hepatic vein access can lead to severe intra-abdominal bleeding. . However, such bleeding complications had been reported primarily during the early period when this procedure was newly introduced, ${ }^{10}$ and reports from centers with relatively good experience in performing this procedure have demonstrated that most procedures can now be performed with minimal bleeding complications. ${ }^{11,12}$ In both patients reported in this paper, ultrasonographically guided transhepatic puncture techniques were performed by highly experienced interventional radiologists, and no bleeding complications were noted. 3) Because the liver moves up and down with respiration, respiratory movement-induced lead dislodgement and/or fracture can occur between the puncture site on the abdominal wall and the liver surface. Therefore, the leads must be firmly fixed and require sufficient redundancy. Moreover, a long suture sleeve should be used and fixed as deeply as possible for protection of the part of the lead that undergoes stress secondary to respiration-induced liver motion.

In conclusion, CIED lead implantation through the hepatic vein can be considered a useful treatment option for selected patients in whom central venous access is difficult/impossible to obtain.

\section{References}

1) Laub GW, Olivencia-Yurvati AH, Muralidharan S, Morse D, Pollock SB, Adkins M, McGrath LB. Permanent transfemoral pacemaker insertion after repair of congenital heart disease. Ann Thorac Surg. 1991;52:148-150.

2) Goto Y, Abe T, Sekine S, Iijima K, Kondoh K, Matsukawa M. Transiliac vein approach to a permanent pacemaker implantation after aortic valve reoperation. Pacing Clin Electrophysiol. 1998;21:1170-1171.

3) Ellestad MH, French J. Iliac vein approach to permanent pacemaker implantation. Pacing Clin Electrophysiol.
1989;12:1030-1033.

4) West JN, Shearmann CP, Gammage MD. Permanent pacemaker positioning via the inferior vena cava in a case of single ventricle with loss of right atrial-vena cava continuity. Pacing Clin Electrophysiol. 1993;16:1753-1755.

5) Goktekin O, Besoglu Y, Dogan SM, Birdane A, Unalir A, Gorenek B, Kudaiberdieva G, Cavusoglu Y, Ata N, Aslan R, Timuralp B. Permanent pacemaker lead implantation via azygous vein in a patient with silent superior vena cava syndrome. Int J Cardiol. 2007;117:e4-6.

6) Fishberger SB, Camunas J, Rodriguez-Fernandez H, Sommer RJ. Permanent pacemaker lead implantation via the transhepatic route. Pacing Clin Electrophysiol. 1996;19:1124-1125.

7) Qureshi AM, Prieto LR, Bradley-Skelton S, Latson LA. Complications related to transhepatic venous access in the catheterization laboratory--a single center 12-year experience of 124 procedures. Catheter Cardiovasc Interv. 2014;84:94-100.

8) Cui K, Feng Y, Li X, Fang Y. Percutaneous transhepatic venous access for ICD implantation in a patient with Ebstein's anomaly with ventricular tachycardia post-Glenn operation. J Cardiovasc Electrophysiol. 2013;24:832-833.

9) Erenberg FG, Shim D, Beekman RH, 3rd. Intraperitoneal hemorrhage associated with transhepatic cardiac catheterization: a report of two cases. Cathet Cardiovasc Diagn. 1998;43:177-178.

10) McLeod KA, Houston AB, Richens T, Wilson N. Transhepatic approach for cardiac catheterisation in children: initial experience. Heart. 1999;82:694-696.

11) Johnston TA, Donnelly LF, Frush DP, O'Laughlin MP. Transhepatic catheterization using ultrasound-guided access. Pediatr Cardiol. 2003;24:393-396.

12) Qureshi AM, Rhodes JF, Appachi E, Mumtaz MA, Duncan BW, Asnes J, Radavansky P, Latson LA. Transhepatic Broviac catheter placement for long-term central venous access in critically ill children with complex congenital heart disease. Pediatr Crit Care Med. 2007;8:248-253. 